\title{
Two-Gluon Correlations in Heavy-Light Ion Collisions
}

\author{
Douglas E. Wertepny \\ Department of Physics, The Ohio State University, \\ Columbus, OH 43210, USA
}

\begin{abstract}
We derive the cross-section for two-gluon production in heavy-light ion collisions in the saturation/Color Glass Condensate framework. This calculation includes saturation effects to all orders in one of the nuclei (heavy ion) along with a single saturation correction in the projectile (light ion). The calculation of the correlation function predicts (qualitatively) two identical ridge-like correlations, near- and away-side. This prediction was later supported by experimental findings in p+A collisions at the LHC. Concentrating on the energy and geometry dependence of the correlation functions we find that the correlation function is nearly center-of-mass energy independent. The geometry dependence of the correlation function leads to an enhancement of near- and away-side correlations for the tip-on-tip $\mathrm{U}+\mathrm{U}$ collisions when compared with side-on-side $\mathrm{U}+\mathrm{U}$ collisions, an exactly opposite behavior from the correlations generated by the elliptic flow of the quark-gluon plasma.
\end{abstract}

Keywords: Color Glass Condensate, rapidity correlations, the ridge

\section{Introduction}

This proceeding contribution is based on the papers [1] and [2].

Two-particle correlations which are long range in rapidity and narrowly peaked around $\Delta \phi=0$, often referred to as the "ridge," were seen at STAR in Au+Au collisions [3]. Since then these types of correlations have been seen in other $\mathrm{A}+\mathrm{A}$ along with $\mathrm{p}+\mathrm{A}$ and $\mathrm{p}+\mathrm{p}$ collisions. Due to causality, correlations which are long-range in rapidity must originate from early-time dynamics. We model the two-particle correlations as two-gluon correlations, anticipating that the correlations between these gluons leave their signature on the final state hadrons. At early times gluon production can be modeled through the saturation/color glass condensate (CGC) framework.

Due to technical constraints we cannot calculate the correlation for the general case of A+A collisions. Instead we calculate the two-gluon correlation function for a heavy-light ion collision: we take into account all orders of saturation effects in the target (the heavy ion) and only a single saturation correction in the projectile (the light ion). This calculation could be applied to $\mathrm{Cu}+\mathrm{Au}$ collisions or even $\mathrm{p}+\mathrm{Pb}$ collisions for high multiplicity collisions.

\section{The Correlation Function in the CGC Framework}

The definition of the correlation function is shown in (1) and was normalized, using the factor $\mathcal{N}$, such that it was zero for the case where there were no correlations. The cross-sections were calculated in the saturation framework;

Email address: $\quad$ wertepny.1@osu.edu (Douglas E. Wertepny) 
while the single-gluon production cross-section was known before, the two-gluon production cross-section was an original calculation.

$$
C\left(\boldsymbol{k}_{1}, y_{1}, \boldsymbol{k}_{2}, y_{2}\right)=\mathcal{N} \frac{\frac{d \sigma}{d^{2} k_{1} d y_{1} d^{2} k_{2} d y_{2}}}{\frac{d \sigma}{d^{2} k_{1} d y_{1}} \frac{d \sigma}{d^{2} k_{2} d y_{2}}}-1
$$

We include a single saturation correction in the light projectile which enhances the probability of independent gluon emission from different nucleons, modeled as valence quarks. Since the collision takes place at high energies the quantum fluctuations of the projectile happen over a much longer time scale than the interaction. The quarks emit gluon, either before or after the interaction with the target nucleus due to the separation of light-cone time scales. The interaction between the projectile and the target is modeled as a shock-wave in the light-cone gauge $\left(A^{+}=0\right)$, where the projectile is moving in the "+" light-cone direction. This required calculating two classes of diagrams, which we call "separated" and "crossed" respectively, shown in Fig. 1. A detailed calculation is presented in [1].
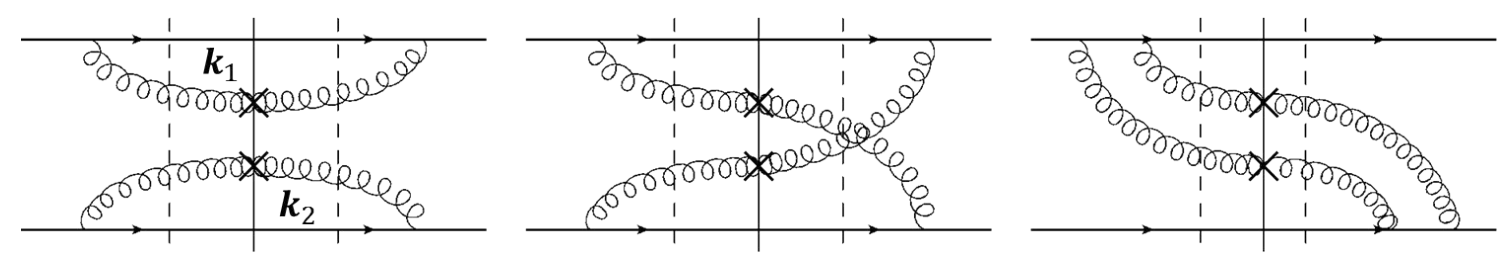

Figure 1. Classes of diagrams calculated in order to arrive at the correlation function: The diagram on the left is an example of a "separated" diagram. The two on the right are examples of "crossed" diagrams. The dashed lines represents the shock-wave interaction with the target.

The calculation yields a correlation function which is symmetric under reflection of the transverse momentum of one of the gluons, $\boldsymbol{k}_{\mathbf{2}} \rightarrow-\boldsymbol{k}_{\mathbf{2}}$, and under the momentum interchange $\boldsymbol{k}_{\mathbf{1}} \leftrightarrow \boldsymbol{k}_{2}$. This results in a correlation that is symmetric in azimuthal angle, giving only even Fourier harmonics in the correlation. Furthermore there are identical enhancements on the near-side $(\Delta \phi=0)$ and the away-side $(\Delta \phi=\pi)$. A toy model that captures the qualitative behavior of the correlation function is shown in Fig. 2. After this result had been originally published in [1], results from ALICE [4] for $\mathrm{p}+\mathrm{Pb}$ collisions showed a near-side and away-side ridge.

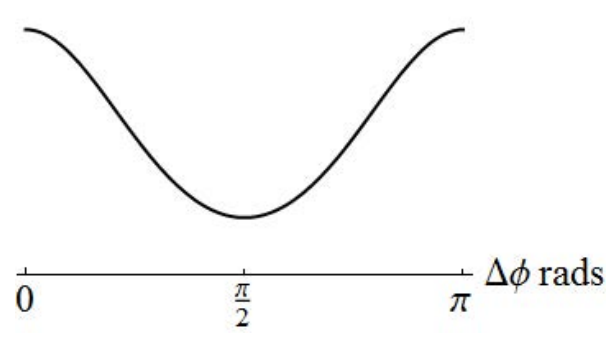

Figure 2. Toy-model for the correlation as a function of azimuthal angle.

In order to get an estimate of the energy dependence of the correlation, one needs the energy dependence of the corresponding cross-sections. While much work has been done on the subject for single gluon production, not much has been done for two-gluon production. In order to get an estimate we used the Gaussian truncation approximation $[5,6]$. Using this approximation we related terms in the two-gluon production cross-section to known solutions for the BK evolution equation, to find the dependence of the correlation function on the center-of-mass energy of the collisions. A detailed derivation is presented in [2] and the results are listed below.

$$
\frac{d \sigma}{d^{2} k d y} \propto e^{\lambda\left(1+2 i v_{0}\right) Y / 2} \quad \frac{d \sigma}{d^{2} k_{1} d y_{1} d^{2} k_{2} d y_{2}} \propto e^{\lambda\left(1+2 i v_{0}\right) Y}
$$


where $\lambda=0.2 \div 0.3$ and $v_{0}=-0.1275 i$.

We can see that when we plug these results into (1) the energy dependence cancels and we are left with an energy independent result for the correlation function.

\section{Discriminating Between Initial State CGC Correlations and Hydrodynamic Flow}

a)

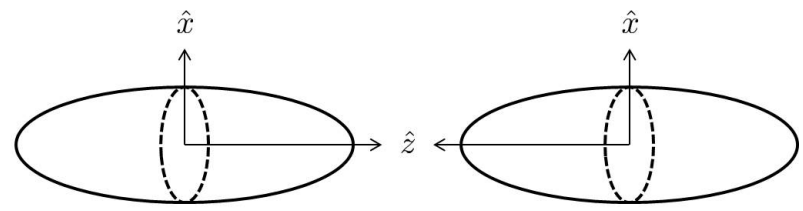

b)

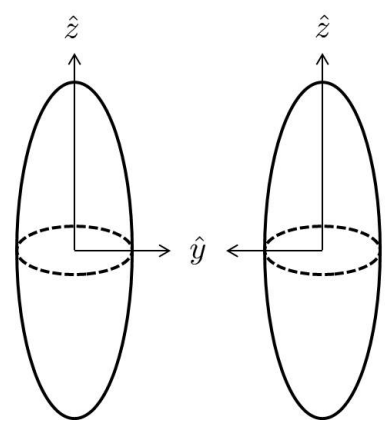

Figure 3. Geometry of the collision types: a) tip-on-tip collision, b) side-on-side collision

The ridge caused by CGC effects is directly related to the saturation scale. This introduces a geometry dependence to the CGC correlations, as the saturation scale, $Q_{s}$, is directly related to the nuclear profile function, $T(\boldsymbol{b}): Q_{s}^{2} \propto T(\boldsymbol{b})$. This is different from the geometric dependence of hydrodynamics which depends on eccentricity of the initial overlap region of the two nuclei, due to the fact that this sets the amount of elliptic flow. This allows us to differentiate the geometrical effects of hydrodynamics from the CGC effects, especially in the collision of two non-spherical nuclei, such as $\mathrm{U}+\mathrm{U}$ collisions. To get an estimate of the effect geometry has on the correlation function we took the ratio of the correlation functions associated with two different geometries for $\mathrm{U}+\mathrm{U}$ collisions, tip-on-tip and side-on-side shown in Fig. 3. For simplicity we modeled the uranium nucleus as a prolate ellipsoid with nucleon number density,

$$
\rho(\overrightarrow{\boldsymbol{r}})=\rho_{0} \exp \left[-\frac{x^{2}}{R^{2}}-\frac{y^{2}}{R^{2}}-\frac{\lambda^{2}}{R^{2}} z^{2}\right]
$$

and calculated the nuclear profile functions for the two different geometries:

$$
\begin{gathered}
T_{\text {tip-on-tip }}(\boldsymbol{b})=\sqrt{\pi} \frac{R}{\lambda} \rho_{0} \exp \left[-\frac{b^{2}}{R^{2}}\right] \\
T_{\text {side-on-side }}(\boldsymbol{b})=\sqrt{\pi} R \rho_{0} \exp \left[-\frac{x^{2}}{R^{2}}-\frac{\lambda^{2}}{R^{2}} z^{2}\right]
\end{gathered}
$$

where $\lambda=0.79$ and is related to the ellipticity of the ellipsoid.

We expanded the correlation function to lowest order in saturation in both the target and the projectile, which corresponds to dilute-dilute collisions and is a valid approximation for $\mathrm{U}+\mathrm{U}$ collisions in the large- $k_{T}$ regime. All of the kinematic factors in the ratio canceled out and we were left with a result depending only on the saturation scale, which in turn is related to the nuclear profile functions. We get 


$$
\frac{\left.C_{\text {tip-on-tip }}\left(\boldsymbol{k}_{1}, y_{1}, \boldsymbol{k}_{2}, y_{2}\right)\right|_{L O}}{\left.C_{\text {side-on-side }}\left(\boldsymbol{k}_{1}, y_{1}, \boldsymbol{k}_{2}, y_{2}\right)\right|_{L O}}=\frac{1}{\lambda} \approx 1.26
$$

We end up with an enhancement of approximately 1.26 for tip-on-tip as opposed to side-on-side collisions. This is significantly different then the result one would get from a hydrodynamic calculation, as side-on-side collisions would dominate due to the fact that the correlation function depends on the elliptic flow which, in turn, is tied to the ellipticity of the overlap region. This gives a clear and measurable distinction between CGC dynamics and hydrodynamic effects.

\section{Conclusions}

Here we have summarized some of the results of the papers [1] and [2]. We modeled two-particle correlations in heavy-light ion collisions to get a better understanding of the ridge through the CGC formalism. We calculated the two-gluon correlation function and found it to have symmetric near-side and away-side ridges. Furthermore we showed that it was nearly center-of-mass energy independent. We found an enhancement in the correlation strength in $\mathrm{U}+\mathrm{U}$ collisions for tip-on-tip geometry as opposed to side-on-side, which is the opposite of what is expected from elliptic flow. This gives a mechanism for distinguishing between the effects of hydrodynamic elliptic flow and the CGC dynamics.

\section{Acknowledgments}

This material is based upon work supported by the U.S. Department of Energy, Office of Science, Office of Nuclear Physics under Award Number DE-SC0004286.

\section{References}

[1] YV Kovchegov, DE Wertepny, Long-Range Rapidity Correlations in Heavy- Light Ion Collisions, Nuclear Physics A (2013), [arXiv:1212.1195].

[2] YV Kovchegov, DE Wertepny, Two-Gluon Correlations in Heavy-Light Ion Collisions: Energy and Geometry Dependence, IR Divergences, and kT- Factorization, Nuclear Physics A (2014), [arXiv:1310.6701].

[3] STAR Collaboration Collaboration, B. Abelev et. al., Long range rapidity correlations and jet production in high energy nuclear collisions, Phys.Rev. C80 (2009) 064912, [arXiv:0909.0191].

[4] ALICE Collaboration Collaboration, B. Abelev et. al., Long-range angular correlations on the near and away side in p-Pb collisions at $\operatorname{sqrt}(\mathrm{sNN})=5.02 \mathrm{TeV},[\operatorname{arXiv}: 1212.2001]$.

[5] Y. V. Kovchegov, J. Kuokkanen, K. Rummukainen, and H. Weigert, Subleading- $N_{c}$ corrections in non-linear small-x evolution, Nucl. Phys. A823 (2009) 47-82, [arXiv:0812.3238].

[6] A. Dumitru, J. Jalilian-Marian, T. Lappi, B. Schenke, and R. Venugopalan, Renormalization group evolution of multi-gluon correlators in high energy QCD, Phys.Lett. B706 (2011) 219-224, [arXiv:1108.4764]. 